\title{
Deep Diving into BitTorrent Locality
}

\author{
[Please cite the IEEE INFOCOM'11 version of this paper]
}

\author{
Ruben Cuevas \\ Univ. Carlos III de Madrid \\ rcuevas@it.uc3m.es
}

\author{
Nikolaos Laoutaris \\ Telefonica Research \\ nikos@tid.es
}

\author{
Xiaoyuan Yang \\ Telefonica Research \\ yxiao@tid.es
}

\author{
Georgos Siganos \\ Telefonica Research \\ georgos@tid.es
}

\author{
Pablo Rodriguez \\ Telefonica Research \\ pablorr@tid.es
}

\begin{abstract}
A substantial amount of work has recently gone into localizing BitTorrent traffic within an ISP in order to avoid excessive and often times unnecessary transit costs. Several architectures and systems have been proposed and the initial results from specific ISPs and a few torrents have been encouraging. In this work we attempt to deepen and scale our understanding of locality and its potential. Looking at specific ISPs, we consider tens of thousands of concurrent torrents, and thus capture ISP-wide implications that cannot be appreciated by looking at only a handful of torrents. Secondly, we go beyond individual case studies and present results for the top 100 ISPs in terms of number of users represented in our dataset of up to $40 \mathrm{~K}$ torrents involving more than $3.9 \mathrm{M}$ concurrent peers and more than $20 \mathrm{M}$ in the course of a day spread in $11 \mathrm{~K}$ ASes. We develop scalable methodologies that permit us to process this huge dataset and answer questions such as: "what is the minimum and the maximum transit traffic reduction across hundreds of ISPs?", "what are the win-win boundaries for ISPs and their users?", "what is the maximum amount of transit traffic that can be localized without requiring finegrained control of inter-AS overlay connections?", "what is the impact to transit traffic from upgrades of residential broadband speeds?".
\end{abstract}

\section{INTRODUCTION}

Most design choices in P2P applications are dictated by end user performance and implementation simplicity. Bootstrapping is one such example: a new node joins a P2P overlay by connecting to a Random set of neighbors. This simple process provides fault tolerance and load balancing to end users and implementation simplicity to developers. Its downside, however, is that it is completely oblivious to the requirements and operating constraints of ISPs and thus it often ends up causing serious problems such as increasing the transit costs, worsening the congestion of unpaid peer- ing links [18, and expediting the upgrade of DSLAMs. Therefore, several ISPs have allegedly started rate limiting or blocking P2P traffic [9]. In response, P2P applications have tried to conceal and evade discriminatory treatment by using dynamic ports and protocol encryption.

Much of this tension can be avoided by biasing the overlay construction of $\mathrm{P} 2 \mathrm{P}$ towards Locality. It is known that geographic proximity often correlates with overlap of consumption patterns 14 and thus bootstrapping $\mathrm{P} 2 \mathrm{P}$ users with other nearby ones can confine P2P traffic within ISPs instead of letting it spill to other domains over expensive transit links. This simple idea has received much attention lately since it is generic and thus can be applied to a variety of $\mathrm{P} 2 \mathrm{P}$ applications independently of their internal logic (scheduling, routing, etc.). Systems like $\mathrm{P} 4 \mathrm{P}$ [26] and $\mathrm{ONO}$ [6] have been proposed for localizing the traffic of the BitTorrent file sharing protocol [7]. $\mathrm{P} 4 \mathrm{P}$ proposes bilateral cooperation between ISPs and P2P applications, whereas ONO is a client-side solution that does not involve the ISP.

Despite the interesting architectures and systems that have already been proposed, we believe that we still stand on preliminary ground in terms of our understanding of this technology. Although the main ideas are straightforward, their implications are quite the opposite, for several reasons. First, different torrents can have quite diverse demographics: a blockbuster movie has peers around the world and thus can create much more transit traffic than a local TV show whose peers are mostly within the same country/ISP, especially if language gets in the way. Predicting the ISP-wide transit traffic due to $\mathrm{P} 2 \mathrm{P}$ amounts to understanding the demographics of thousands of different torrents downloaded in parallel by all the customers. Things become even more complicated in the case of the BitTorrent protocol whose free-riding avoidance scheme makes peers exchange traffic predominately with other peers of similar speed [17. Thus even if two ISPs have similar demo- 
graphic composition, the fact that they offer different access speeds can have a quite pronounced impact on the amount of transit traffic that they see. The combined effect of demographics and access speeds makes it risky to generalize observations derived from a particular ISP and few individual torrents.

\section{OUR CONTRIBUTIONS}

Our works provides detailed case studies under representative ISP-wide workloads as well as holistic views across multiple (hundreds) of ISPs. In all cases we demand that the input be as representative as possible (demographics and speed of different ISPs) and the methodology be scalable without sacrificing essential BitTorrent mechanisms like the unchoke algorithm, the least replicated first chunk selection policy, and the effect of seeders. We collected representative input data by scraping up to $100 \mathrm{~K}$ of torrents of which at least $40 \mathrm{~K}$ had active clients from Mininova and Piratebay, the two most most popular torrent hosting sites in the world according to the Alexa Ranking. We then queried the involved trackers to construct a map of BitTorrent demand demographics of up to $3.9 \mathrm{M}$ concurrent users and more than $21 \mathrm{M}$ total users over the course of a day, spread over $11 \mathrm{~K}$ ISPs. For all those ISPs we obtained speeds from a commercial speed-test service [2] and from the iPlane project [19].

Our datasets are too big to conduct emulation or simulation studies. To process them, we employ two scalable methodologies: a probabilistic one for deriving speed-agnostic upper and lower bounds on the number of piece exchanges that can be localized within an ISP given its demand demographics and a more accurate deterministic one that estimates the resulting traffic matrix taking into consideration the speeds of different ISPs. The probabilistic technique allows us to scale our evaluation up to as many ISPs as we like (we report on the 100 largest ones) whereas the deterministic one allows us to zoom in into particular ISPs and refine our estimation of transit traffic and end-user QoS. With these two tools, we study the performance of a rather broad family of overlay construction mechanisms that includes: Locality Only If Faster, (LOIF), an end-user QoS preserving overlay that switches remote neighbors for locals only when the latter are faster, and Locality, a simple policy that maximizes transit savings by switching as many remote neighbors as possible with local ones, independently of relative speed.

Summary of results: We shed light to several yet unanswered questions about BitTorrent traffic. Specifically:

- We use the demand demographics of the 100 largest ISPs from our dataset to derive speed agnostic upper and lower bounds on the number of chunk exchanges that can be kept local. In half of the ISPs, Locality keeps at least $42 \%$ and up to $72 \%$ of chunks internal, whereas Random can go from less than $1 \%$ up to $10 \%$.

Next we focus on the three largest US and the three largest European ISPs in our dataset and derive their traffic matrices using both demographic and speed information. These detailed case studies reveal the following:

- LOIF preserves the QoS of users and reduces the transit traffic of fast ISPs by around 30\% compared to Random. In slower ISPs the savings are around $10 \%$.

- Locality achieves transit traffic reductions that peak at around $55 \%$ in most of the ISPs that we considered. The resulting penalty on user download rates is typically less than $6 \%$.

- The barrier on transit traffic reduction is set by "unlocalizable" torrents, i.e., torrents with one or very few nodes inside an ISP. Such torrents account for around $90 \%$ of transit traffic under Locality and are requested by few users of an ISP $(\sim 10 \%)$. In a sense, the majority of users is subsidizing the transit costs incurred by the few users with a taste for unlocalizable torrents.

- By limiting the number of allowed inter-AS overlay links per client huge reductions of transit (>95\%) are possible. The resulting median penalty is around $20 \%$ but users on "unlocalizable" torrents incur huge reduction of QoS (99\%).

- Finally, we show that, contrary to popular belief, increasing the speed of access connections does not necessarily keep more traffic local as it might bring an ISP within unchoke distance from other fast ISPs which previously did not send it traffic.

Overall our results show that there is great potential from locality for both ISPs and users but there also exist some cases in which locality needs to be approached with caution. Cashing in this potential in practice is a non-trivial matter, but seems to be worthy of further investigation.

The remainder of the article is structured as follows. In Sect. 3we derive upper and lower bounds on the number of localized unchokes under Random and Locality overlays, independently of ISP speed distributions. In Sect. 4 we present our measurement study of BitTorrent demographics. We also define a metric for explaining the performance of Random when factoring in real speed distributions across ISPs. In Sect. 5] we present a methodology for estimating BitTorrent traffic matrices 
and in Sect. 66 we define the family of overlay construction policies that we use later in our study. Sect. 7 characterizes the win-win situations and the tradeoffs between ISPs and users under different locality policies. In Sect. 8 we present a validation prototype for studying locality using live torrents and factoring in network bottlenecks. In Sect. 9 we look at related work and we conclude in Sect. 10 .

\section{WHY NOT A RANDOM OVERLAY?}

Our goal in this section is to understand the cases in which a Random selection of neighbors localizes traffic well, and the ones in which it fails thereby creating the need for locality-biased neighbor selection. To do so we first need to understand the stratification effect [1] arising due to the unchoke algorithm [7] used by BitTorrent to combat free-riding. According to this algorithm, a node monitors the download rates from other peers and "unchokes" the $k$ peers (typically $4-5$ ) that have provided the highest rates over the previous $20 \mathrm{sec}$ interval. These peers are allowed to fetch missing chunks from the local node over the next $10 \mathrm{sec}$ interval. Therefore, as long as there are chunks to be exchanged between neighbors (LRF chunk selection works towards that [7), peers tend to stratify and communicate predominantly with other peers of similar speed.

In this section, we employ probabilistic techniques to help us build some basic intuition on the consequences of stratification on inter-domain traffic. We focus on a single ISP $A$ and torrent $T$ and analyze the conditions under which Random localizes sufficiently within $A$ the traffic due to $T$. In Sect. 4 we will examine the effects from multiple torrents with different demographics as well as the effect of speed differences between ISPs. In Sect. 5 we will go into an even more accurate model that captures more precisely the unchoke behavior of leechers (including optimistic unchokes) as well as the different behavior of seeders. We will also discuss the impacts of torrents that are not in steady-state and develop a model for them (Appendix C).

\subsection{Sparse mode - the easy case for Random}

Let $V(T)$ denote the set of BitTorrent nodes participating in $T$, and $V(A, T) \subseteq V(T)$ the subset that belongs to ISP $A$. We say that ISP $A$ is on sparse mode with respect to torrent $T$ if the nodes outside $A$ that participate in $T$ have very dissimilar speeds with nodes that are within $A$. In this case, because of stratification, local nodes of $A$ will talk exclusively to each other irrespectively of other remote nodes in their neighborhood. Then to confine all unchokes within $A$, each local node needs to know at least $k$ other local neighbors. If $W$ denotes the size of a neighborhood (40 upon bootstrap and growing later with incoming connections), then for Random to localize all traffic it has to be that a ran- dom draw of $W$ out of the total $|V(T)|-1$ (-1 to exclude the node that is selecting) nodes yields at least $k$ local ones. The probability of getting $x$ "successes" (i.e., local nodes) when drawing randomly $W$ samples from a pool of $|V(T)|-1$ items, out of which $|V(A, T)|-1$ are "successes" is given by the Hyper-Geometric distribution HyperGeo $(x,|V(T)|-1,|V(A, T)|-1, W)[10$. Thus the expected number of localized unchokes is

$\sum_{x=0}^{\min (|V(A, T)|-1, W)} \min (x, k) \cdot \operatorname{HyperGeo}(x,|V(T)|-1,|V(A, T)|-1, W)$

Taking the mean value of the distribution we can write a condition for Random to localize well in sparse mode:

$$
\frac{W \cdot(|V(A, T)|-1)}{|V(T)|-1} \geq k
$$

\subsection{Dense mode - things getting harder}

ISP $A$ is on dense mode with respect to $T$ if the remote nodes participating in $T$ have similar speeds to the nodes of $A$. In this case stratification does not automatically localize traffic inside $A$. From the standpoint of the unchoke algorithm, both local and remote nodes look equally good and thus the number of localized unchokes depends on their ratio in the neighborhood. Thus, although in sparse mode a random draw yielding $x \leq k$ local nodes would keep all $x$ unchokes local, in dense mode it keeps only $k \cdot x / W$ of them local in expectation. To get the expected number of localized unchokes in dense mode we have to substitute $\min (x, k)$ with $k \cdot x / W$ in Eq. (1).

\subsection{The promise of Locality}

Let's now consider Locality, an omniscient overlay construction mechanism that knows all local nodes and thus constructs highly localized neighborhoods by providing each node with as many local neighbors as possible, padding with additional remote ones only if the locals are less than $W$. Then in sparse mode Locality localize all unchokes as long as $|V(A, T)|-1 \geq k$, which is a much easier condition to satisfy than the one of Eq. (2), else it localizes only $|V(A, T)|-1$. In dense mode Locality localizes all unchokes as long as $|V(A, T)|-1 \geq W$.

\subsection{Locality gains are higher in dense mode}

Overall Random localizes sufficiently in sparse mode as long as it can get a small number of local nodes in each neighborhood. In dense mode things become more challenging as it no longer suffices to guarantee a small threshold of locals but instead Random has to have a strong majority of locals in each neighborhood. In both modes, Locality has to satisfy easier conditions to localize the same number of unchokes. Further, we can 
actually prove that the improvement factor of Locality over Random in terms of the number of localized unchokes is higher in dense mode than in sparse mode. We consider only the case with $|V(A, T)|-1 \geq k$ and $|V(T)|-1 \geq W$ (the other ones can be worked out similarly). Based on the previous analysis we get that the expected improvement factor in sparse mode is:

$$
\frac{k}{W \cdot \frac{|V(A, T)|-1}{|V(T)|-1}}
$$

In dense mode for $|V(A, T)|-1 \geq W$ the improvement factor is:

$$
\frac{k}{k \cdot \frac{|V(A, T)|-1}{|V(T)|-1}}
$$

which is greater than Eq. (3) since $W>k$. For $|V(A, T)|-1<W$ the improvement factor is:

$$
\frac{k \cdot \frac{|V(A, T)|-1}{W}}{k \cdot \frac{|V(A, T)|-1}{|V(T)|-1}}=\frac{|V(T)|-1}{W}
$$

which can be checked to be greater than Eq. (3) for the case with $|V(A, T)|-1 \geq k$. In Sect. 4.3 we will use measurement to determine the ISP-wide improvement factor.

\section{DEMOGRAPHICS OF BITTORRENT}

We conducted a large measurement study of BitTorrent demand demographics. We begin with a presentation of our measurement methodology and then use the obtained demographics to derive upper and lower bounds on the number of localized regular unchokes under Random and Locality. At the end of the section we incorporate the effect of speed differences among ISPs and show that it is non trivial to predict what happens to the transit traffic of an ISP when it upgrades the speed of its residential accesses.

\subsection{Measurement methodology}

We developed a custom BitTorrent crawler that obtains a snapshot of the IP addresses of all the clients participating in a set of torrents that are provided as input. In Table 1 we present the different sets of torrents used in our study. Our crawler first scrapes a torrent indexing site to obtain .torrent meta information files. From them it obtains the addresses of the corresponding trackers. It queries repeatedly the trackers and uses the gossip protocol implemented in the latest versions of BitTorrent to obtain all the IP addresses of clients participating in each torrent. The gathered IP addresses are mapped to ISPs and countries using the MaxMind database [1. The crawler also obtains the number of seeders and leechers in each torrent. Crawling an individual torrent takes less than 2 minutes. Thus we get a pretty accurate "snapshot" of

\begin{tabular}{ccccc}
\hline Set name & Source & Torrents & \# IPs & \# ISPs \\
\hline mn40K & Mininova & latest 40K & $3.9 \mathrm{M}$ & $10.4 \mathrm{~K}$ \\
mn3K & Mininova & latest 3K & $17.4 \mathrm{M}$ & $10.5 \mathrm{~K}$ \\
pb600 & Piratebay & 600 most popular & $21.9 \mathrm{M}$ & $11.1 \mathrm{~K}$ \\
\hline
\end{tabular}

Table 1: Torrent sets collected in the period Aug-Oct 2009. For mn40K we collected three versions, with one week in between them. We actually crawled $100 \mathrm{~K}$ torrents but only around $40 \mathrm{~K}$ had peers. For $\mathrm{mn} 3 \mathrm{~K}$ and $\mathrm{pb} 600$ we repeated the crawl every hour for one day. The \#IPs and \#ISPs for mn40K are per snapshot, whereas for $\mathrm{mn} 3 \mathrm{~K}$ and $\mathrm{pb} 600$ are daily totals.
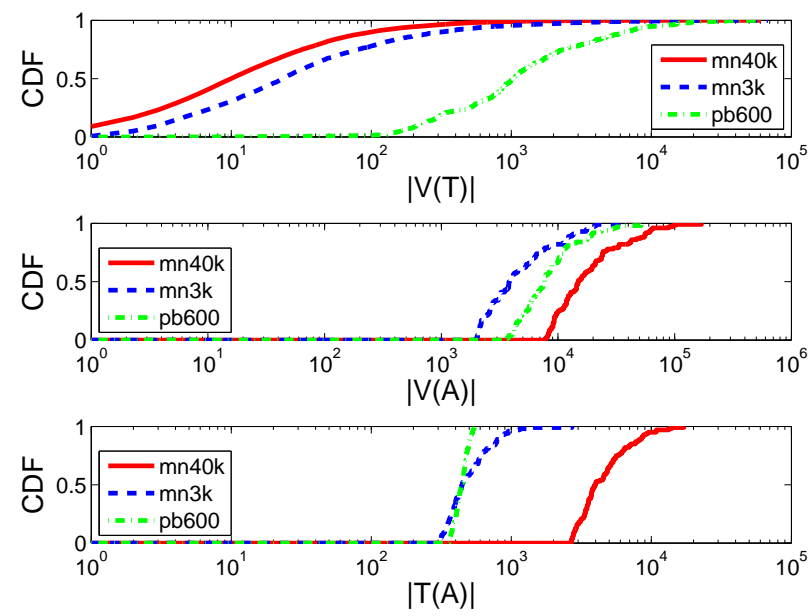

Figure 1: Summary statistics for the measured BitTorrent demographics. Cdfs for: $|V(T)|$, the number of clients in a torrent, $|V(A)|$, the total number of clients in an ISP across all its torrents, and $|T(A)|$, the number of distinct torrents requested by the clients of an ISP.

each torrent, i.e., we are sure that the obtained IPs are indeed present at the same time. The time difference between the first and last crawled torrent was up to 90 minutes for the largest dataset (mn40K). However, we tracked individual torrent populations and found them to be quite stable across a few hours. Thus our dataset is similar to what we would get if we used a very large number of machines to crawl more torrents in parallel. We specifically wanted to avoid that since it would be a denial of service attack against the hosting sites and the trackers.

\subsection{High level characterization of the dataset}

We use the following definitions. We let $\mathcal{T}$ denote the set of torrents appearing in our measurements and $\mathcal{A}$ the set of ISPs that have clients in any of the torrents of $\mathcal{T}$. We let $T(A)$ denote the set of torrents 

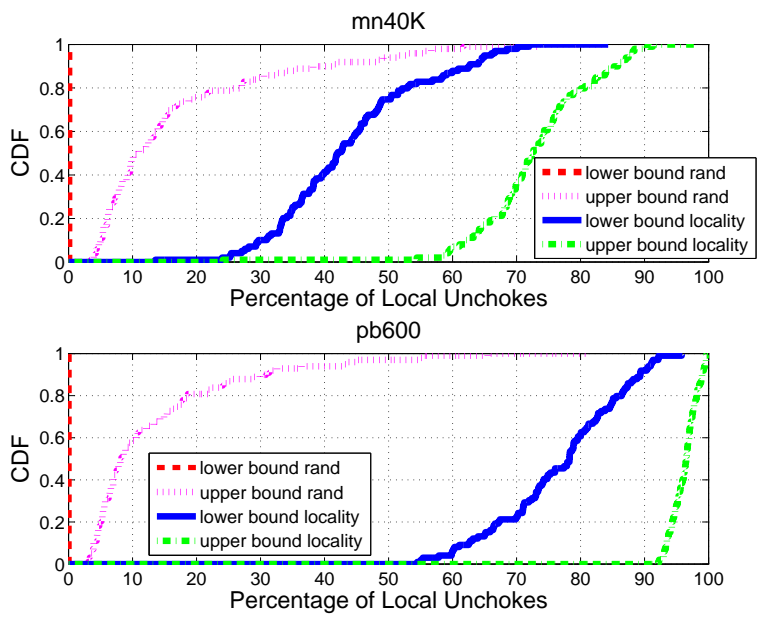

Figure 2: CDF of the upper and lower bound on the number of localized unchokes under Random and Locality for top-100 ISPs in number of clients. Top: mn40K dataset. Bottom: pb600 dataset.

that have at least one active client in $A$, and $V(A)=$ $\bigcup_{T \in T(A)} V(A, T)$ the set of all clients of $A$ participating in any of the torrents $T(A)$. In Fig. 1 we summarize the measured demographics. Some points worth noting:

The largest torrent has approximately $60 \mathrm{~K}$ clients in all three datasets. Looking at the large set, mn40K we see that most torrents are small, as has already been shown [22, 21]. mn3K has relatively bigger torrents since it is a subset of most recent torrents of mn40K, and recency correlates with size. pb600 holds by definition only big torrents.

Looking at the number of peers and torrents per ISP we see that $\mathrm{mn} 40 \mathrm{~K}$ has bigger values which is expected since it is a much bigger dataset than the other two and thus contains more and bigger ISPs (notice that in Table 1 the numbers for $\mathrm{mn} 40 \mathrm{~K}$ are per snapshot, whereas for the other two are aggregates over a day, i.e., totals from 24 snapshots).

\subsection{Speed agnostic bounds for the measured demand demographics}

In Sect. 3 we defined the notions of sparseness and denseness for one ISP and a single torrent and noted that sparseness helps to localize traffic whereas denseness makes it harder. Therefore, by assuming that all the torrents $T(A)$ downloaded in $A$ are concurrently in sparse mode we can get an upper bound on the expected number of unchokes that can be localized by an overlay construction policy for the given demand demographics and any speed distribution among different ISPs. Similarly, by assuming that all torrents are in dense mode we get a lower bound. In Fig. 2 (top) we plot the up- per and lower bound on localized unchokes for Random and Locality for the top-100 ISPs in number of clients in the mn40K dataset (this list includes all major ISPs and amounts for more than $68 \%$ of all IPs in the dataset). These bounds were computed using formula (11) and its corresponding version for dense mode for single torrents and iterating over all $T \in T(A)$ from our demographics dataset adding each contribution with weight $|V(A, T)| / \sum_{T^{\prime} \in T(A)}\left|V\left(A, T^{\prime}\right)\right|$ to capture the relative importance of $T$ for $A$.

The lower bound for Random is very close to 0 . This happens because for the huge majority of torrents, an ISP has only a small minority of the total nodes in the torrent. In dense mode, Random needs to get most of these few locals with a random draw which is an event of very small probability. On the other hand, this small minority of nodes performs much better in sparse mode yielding an upper bound for Random that is at least $10.94 \%$ in half of the top-100 ISPs. Locality has strikingly better performance. Its lower bound is at least $42.35 \%$ and its upper bound $72.51 \%$ in half of the top-100 ISPs. The huge improvement comes from the fact that Locality requires the mere existence of few local nodes in order to keep most unchokes inside an ISP. As noted earlier, the improvement factor is greater in the difficult case (the lower bound goes from 0 to above $42 \%$ in half of the cases) while it is also quite big in the easy case (improvement factor of at least 6.63 in half of the cases).

In Fig. 2 (bottom) we recompute these bounds based on the pb600 dataset. In this case, the upper bound of Random is lower since nodes from the same ISP become an even smaller minority in very large torrents. On the other hand, Locality benefits in terms of both upper and lower bounds. This happens because the bounds for Locality, unlike Random, depend on the absolute rather than the relative number of local nodes, which increases with larger torrents. These bounds paint, to the best of our knowledge, the most extensive picture reported up to now in terms of covered ISPs and torrents of the potential of locality given the constraints set by real demand demographics.

\subsection{Factoring the effect of speed}

The notions of sparseness and denseness have been useful in deriving speed-agnostic performance bounds based on the demand demographics and the overlay construction policy. To refine our analysis and answer more detailed questions we turn our attention now to the effect of speed. We do so through what we call Inherent Localizability.

Let $A(T)$ denote the set of ISPs that have clients in torrent $T$. Let also $U(A)$ denote the uplink speed of nodes in ISP $A$. We focus on the uplink speeds because they are typically the bottleneck in highly asymmetric 

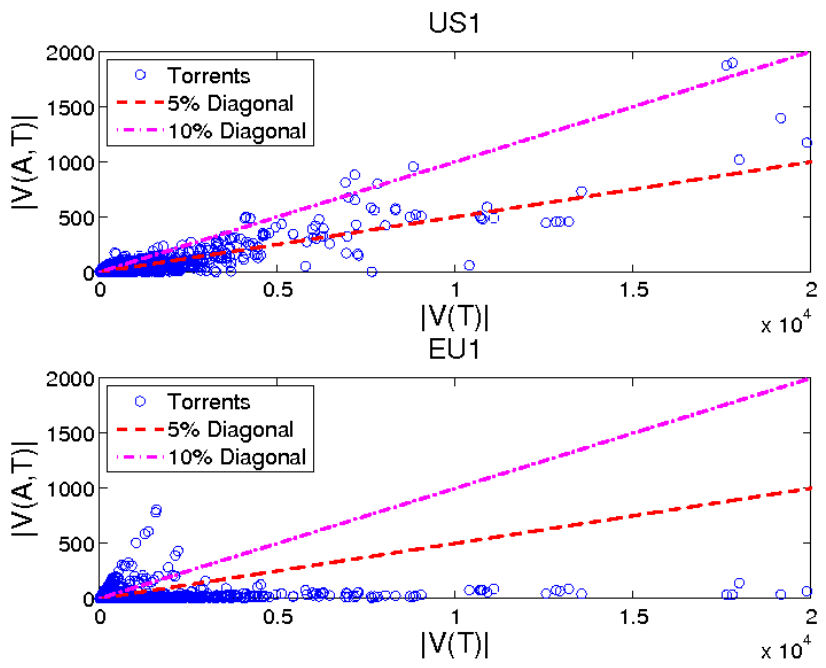

Figure 3: Nodes in ISP A, $|V(A, T)|$, vs. total torrent size, $|V(T)|$, for US1 (top) and EU1 (bottom).

residential broadband accesses [8]. For now it suffices to assume that speeds differ only between ISPs (we relax this in Sect. 5 by considering speed distributions within an ISP). We define the Inherent Localizability $I_{q}(A, T)$ of torrent $T$ in ISP $A$ as follows:

$$
I_{q}(A, T)=\frac{|V(A, T)|}{\sum_{A^{\prime} \in A(T)}\left|V\left(A^{\prime}, T\right)\right| \cdot \mathcal{I}\left(A, A^{\prime}, q\right)},
$$

where, $\mathcal{I}\left(A, A^{\prime}, q\right)=1$ iff $U(A) \cdot(1-q) \leq U\left(A^{\prime}\right) \leq$ $U(A) \cdot(1+q)$, and 0 otherwise. The parameter $q \in[0,1]$, captures the maximum speed difference that still allows a local node of $A$ and a remote node of $A^{\prime}$ to unchoke each other. In reality $q$ can be arbitrarily large since very fast nodes can unchoke much slower ones in the absence of other fast nodes. We use this simple metric here in order to gain a basic intuition into the combined effects of speed and demographics and discard it later in the context of a more accurate albeit more complex model for predicting unchoke decisions in Sect. 5. The inherent localizability $I_{q}(A)$ of ISP $A$ across all its torrents is simply the weighted sum by $|V(A, T)| /|V(A)|$ of its $I_{q}(A, T)$ 's for all torrents it participates in. $I_{q}(A)$ captures the density of $A$ 's nodes in torrents that it shares with other ISPs that have similar speed. Due to stratification, unchokes will take place among those nodes. For Random, $I_{q}(A)$ determines completely its ability to localize unchokes. $I_{q}(A)$ also impacts on Locality. However, Locality's overall performance depends on the absolute number of local peers.

\subsection{Does being faster help in localizing better?}

In this section we use inherent localizibility to study the effect of access speed on the ability of Random to

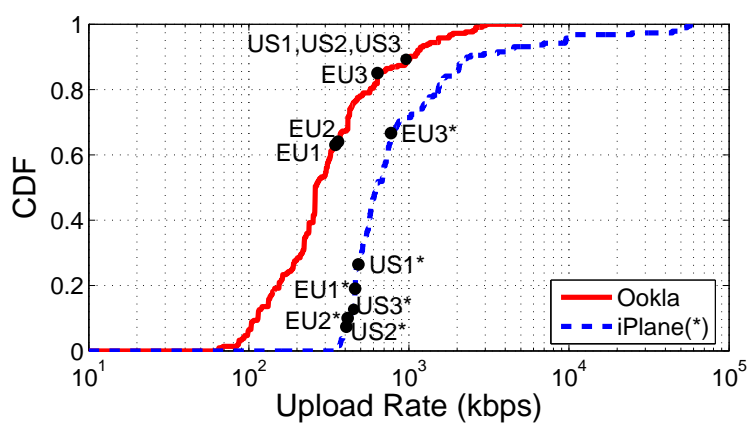

Figure 4: CDF of uplink speeds per country. EU1-EU3, US1-US3 are ISPs studied in Sect. 7.

keep unchokes internally in an ISP. ISPs have a natural interest in this question since on one hand they want to upgrade their residential broadband connections to fiber but on the other hand, they wonder how this will impact their transit and peering traffic. Next we present a case study showing that it is difficult to come up with such predictions without using detailed demographic/speed information and corresponding methodologies to capture their combined effect.

\subsubsection{A European and an American ISP}

Consider the following two ISPs from our dataset mn40K: US1, with the largest population of nodes in America (according to our different datasets) and median upload speed $960 \mathrm{Kbps}$, and EU1, with the largest population of nodes in Europe and median upload speed $347 \mathrm{Kbps}$. In Fig. 3 we plot $|V(A, T)|$ vs. $|V(T)|$ for all $T \in T(A)$ for the two ISPs. A quick glance at the figure reveals that the two ISPs are radically different in terms of demand demographics. Because of the proliferation of English and its English content, US1 is participating in globally popular torrents. In the figure, the US1 torrents that are globally large (high $|V(T)|$ ) have a lot of clients inside US1. Also, torrents that are popular in US1 are also globally popular. In EU1 the picture is very different. The largest torrents inside EU1 are not among the largest global ones, whereas only very few globally popular torrents are also popular inside EU1. This has to do with the fact that EU1 is in a large nonEnglish speaking European country that produces and consumes a lot of local, or locally adapted content.

\subsubsection{The impact of demographics and speed on in- herent localizability}

We will now compute the inherent localizability of EU1 and US1. To do this we need the speeds $U(A)$ for all $A$ that participate in common torrents with the two ISPs. We have obtained these speeds from the Ookla Speedtest service 2]. This data set includes measurements of both upload and download speeds of over 19 

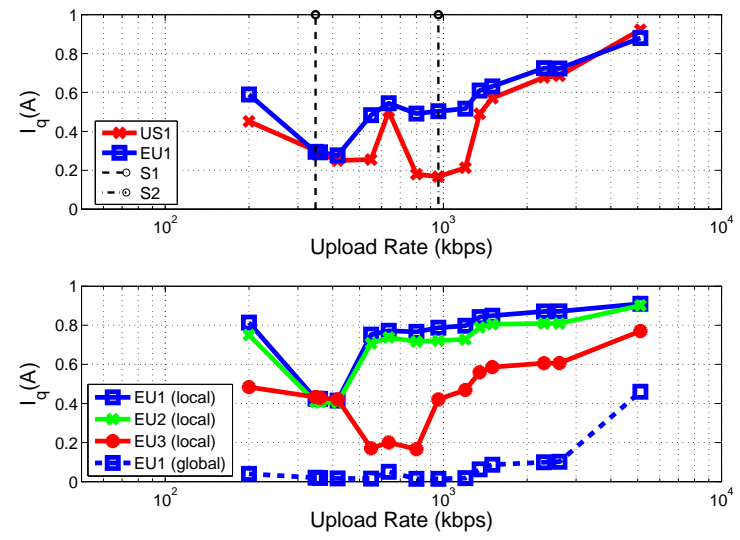

Figure 5: Top: the inherent localizability of US1 and EU1 for different speeds based on all their torrents. Bottom: The inherent localizability of 3 European ISPs based on their 10 most popular local torrents and the $\mathbf{1 0}$ most popular torrents across the entire dataset.

million IP client addresses around the world. In Fig. 4 we plot the cdf of median country speed based on the above dataset. It is interesting to observe that almost $80 \%$ of the countries have similar speeds that are below $610 \mathrm{Kbps}$ where the few remaining ones are sparsely spread in the range from $610 \mathrm{Kbps}$ to $5.11 \mathrm{Mbps}$. We also plot the corresponding cdf from iPlane [19] which we use later for validation.

Using the above demographics and speeds we plot in Fig. 5 (top) the localizability of ISP $A \in\{$ EU1,US1 $\}$ for different $U(A)$, i.e., we plot how the localizability of the two ISPs would change if we changed their speeds while keeping the speeds of all other ISPs fixed. We have assumed $q=0.25$. Results are similar for most $q<0.5$ whereas for larger ones speed starts becoming marginalized because high $q$ 's imply that any node can unchoke any other one. There are two points to keep from this figure. First, the localizability of EU1 is generally higher than that of US1 for the same speed. This means that if the two ISPs had similar speed, then the demographic profile of EU1 depicted earlier in Fig. 3 would lead to a higher inherent localizability since this ISP holds a larger proportion of the content requested by its users. Thus Random would perform better in EU1 than in US1.

A second point to notice is that $I_{0.25}(A)$ is changing non-monotonically with $U(A)$. This happens because the set of remote ISPs and consequently the number of remote clients that can be unchoked by clients of $A$ due to similar speed (within the margins allowed by a given q) changes as we vary the speed of $A$. If the torrents were spread uniformly across all the ISPs, and ISPs had similar size, then due to the sparsification of ISPs on the high speed region (Fig. (4), $I_{0.25}(A)$ would increase monotonically with $U(A)$. The real demographics and sizes of ISPs, though, lead to the depicted nonmonotonic behavior that exhibits only a general trend towards higher intrinsic localizibility with higher local speed. This has important consequences on the expected amount of transit traffic under different speeds. For example, by going from speed $\mathrm{S} 1=347 \mathrm{Kbps}$ to $\mathrm{S} 2$ $=960 \mathrm{Kbps}$, the inherent localizability of EU1 increases from around 0.3 to around 0.5 and as a consequence its transit traffic under Random would decrease as more unchokes would stay inside the ISP. The opposite however happens for US1. Increasing the speed from S1 to $\mathrm{S} 2$ reduces the inherent localizability from 0.3 to 0.2 , effectively increasing the number of unchokes going to remote nodes and thus the transit traffic as well.

\subsubsection{Local and global torrents}

It seems very difficult to devise simple rules of thumb for predicting how $I_{q}(A)$ will change with $U(A)$ without using detailed demographic and speed information as we did earlier. The complication owes to the torrent mix of each ISP, which includes both global and local torrents. Global torrents are those very popular torrents consumed by users around the word. Global torrents are omnipresent in the entire speed range, but since the country speed cdf sparsifies at higher ranges (Fig. 4), fewer of them will be encountered as remote neighbors when an ISP upgrades to such speeds. This leads to more internal unchokes of global torrents, effectively making the inherent localizibility of global torrents a monotonic function of speed.

Local torrents exist at specific ISPs and speed ranges and thus their behavior during speed upgrades is more difficult to predict. For example, an ISP at a French speaking African country will see its unchokes to and from remote ISPs increasing if it upgrades its residential accesses to speeds that bring it near the offered speeds in France, Belgium, and Canada. If it upgrades to even faster speeds though, its remote unchokes will fall since its local users would rather unchoke each other than peers in other countries. In Fig. 5 (top) the localizability of US1 fell at around 1Mbps because it entered the region of many other US ISPs and thus started exchanging unchokes with them for content that although in English, is local to US (local TV, music, etc.). In Fig. 5 (bottom) we compute the inherent localizability of the 10 most popular local torrents in 3 European countries and the corresponding 10 most popular across the entire dataset. The global torrents change monotonically whereas local ones do not. The main point here is that since the interplay between speed and demographics is complicated, an ISP can use our methodology to actually obtain an informed prediction of the impact of planned changes to its residential broadband offerings 
on its transit traffic.

\section{BITTORRENT TRAFFIC MATRICES}

Our analysis up to now has been used for building up a basic intuition about the parameters that affect the performance of Random and Locality. However it has a number of shortcomings. First, it makes the simplifying assumption that nodes whose speeds do not differ more than a multiplicative factor $(1 \pm q)$ unchoke each other. The problem with this assumption is that depending on the speed distribution, there may not be a single $q$ that predicts all the unchokes. Also, the analysis does not capture the behavior of seeders or the optimistic unchokes from leechers. In the next section we develop a more accurate model that addresses all these shortcomings and predicts the actual traffic matrix resulting from a set of torrents. Our objective is to estimate the aggregate amount of traffic routed to an ISP transit link due to the torrents of our demographic datasets of Table 1 .

We start with fast numeric methods that capture the unchoking behavior in steady-state, i.e., when the Least Replicated First (LRF) chunk selection algorithm [7] has equalized the replication degree of different chunks at the various neighborhoods. From that point in time on, we can factor out chunk availability and estimate the established unchokes based only on the uplink speed of nodes. In Appendix C we extend this numeric method to capture also the initial flash-crowd phase of a torrent. The resulting model is much slower in terms of execution time and provides rather limited additional fidelity since the flash crowd phase is known to be relatively short compared to the steady-state phase of sufficiently large downloads (the size of a movie or a software package) $13,16,15$. For this reason we stick to the original more scalable model.

Notice that although experimentation with real clients would provide higher accuracy in predicting the QoS of individual clients, it wouldn't be able to scale to the number of torrents and clients needed for studying the impact of realistic torrent demographics at the ISP level (aggregate traffic in the order of several Gbps). Our scalable numeric methodology targets exactly that while preserving key BitTorrent properties like leecher unchoking (regular and optimistic) and seeding. We validate the accuracy of our methods against real BitTorrent clients in controlled emulation environments (Appendix B) and in the wild with live torrents (Sect.8).

\subsection{Modeling Seeders}

Let $N(s, T) \subseteq$ be the neighborhood of a seeder node $s$ of torrent $T$. Existing seeders typically split their uplink capacity $U(s)$ among their neighbors following one of two possible policies. In the Uniform policy, all neighbors $u \in N(s, T)$ get an equal share $\operatorname{upload}(s, u)=$
$U(s) /|V(s, T)|$. In the Proportional policy, neighbor $u \in N(s, T)$ gets an allotment in proportion to its speed, i.e., upload $(s, u)=U(s) U(u) \mid / \sum_{u^{\prime} \in N(s, T)} U\left(u^{\prime}\right)$.

\subsection{Modeling Leechers}

Estimating the traffic flow among leechers is more involved due to the unchoke algorithm [7]. This reciprocity based matching algorithm of nodes with similar speeds has many of the elements of a $b$-matching problem [5, 11. In Appendix $\mathrm{A}$ we show how to cast the problem of estimating the traffic matrix from a torrent $T$ downloaded by nodes in $V(T)$ as a $b$-matching problem in $V(T)$. We also point to work describing how to get a fast solution (a stable matching $M$ ) for the $b$ matching. $M$ gives us the pairs of nodes that unchoke each other in steady-state. Using the stable matching $M$ and the uplink speeds of nodes, we can compute the expected rate at which a node $v$ uploads to its neighbor $u$ :

$$
\operatorname{upload}(v, u)= \begin{cases}\frac{U(v)}{k+1}, & \text { if }(v, u) \in M \\ \frac{U(v)}{k+1} \cdot \frac{1}{|N(v, T)|-k}, & \text { otherwise }\end{cases}
$$

The first case amounts to neighbors $u$ that are allocated one of $v$ 's $k$ regular unchokes in steady-state. The second case amounts to the remaining neighbors that receive only optimistic unchokes and thus share the single slot that is allocated optimistically 1 In Appendix B we have validate the accuracy of the $b$-matching for estimating unchokes during the two typical phases of a torrent's lifetime [24]. In Sect. 7 we will use the upload rates from the $b$-matching described above and the corresponding seeder bandwidth allocation policies of Sect. 5.1 to compute the amount of BitTorrent traffic crossing inter-AS links. Before that, however, we introduce the overlay construction policies that we will study.

\section{LOCALITY-BIASED OVERLAYS}

Up to now our discussion has been based on a very basic locality biasing overlay construction algorithm, Locality, that provides a node $v$ of $A$ participating in $T$ with $\min (W,|V(A, T)|-1)$ local nodes and pads up to $W$ with randomly chosen remote nodes. In this section we want to generalize Locality so that it can capture the operation of existing overlay construction policies like the ones proposed in [26, 6].

\subsection{A family of locality-biased overlays}

\footnotetext{
${ }^{1}$ It might be the case that in a stable solution node $v$ is matched to less than $k$ others (e.g., because it is of low preference to its neighbors). In such cases we add the unallocated unchoke bandwidth to the optimistic unchoke bandwidth that is evenly spread to choked neighbors.
} 
We refer to the resulting extended family of overlay construction algorithms as $\operatorname{Locality}(\delta, \mu)$. Its operation is as follows. It starts with a neighborhood $N(v, T)$ of $\max (W,|V(T)|-1)$ randomly selected neighbors which are then filtered based on speed comparisons against the set of local nodes $V(A, T) \backslash\{v\}$. These comparisons are modulated by the parameters $\delta, \mu$ as follows. Parameter $\mu$ controls the maximum number of allowed remote (inter-AS) neighbors in $N(v, T)$. If the number of remote nodes in $N(v, T)$ is greater than $\mu$ then a remote node $u$ is substituted by a local $w$ that is not already in the neighborhood until the number of remotes reaches $\mu$. If there are no more local nodes for performing switches then $u$ is taken out of the neighborhood. If the number of remotes in $N(v, T)$ is already below $\mu$, then $u$ is substituted by a not already taken local node $w$ only if $1-\frac{U(w)}{U(u)}<\delta$.

For now we won't concern ourselves with implementation issues as we only use these policies for exploring the transit reduction vs. user QoS tradeoff. We will discuss implementation issues later in Sect. 8 ,

\subsection{Some notable members}

In the evaluation presented in Sect. 7 we will consider some members of the Locality $(\delta, \mu)$ that are of special interest. These include:

- $\delta=0, \mu=\min (W,|V(T)|-1)$ : In this case there is no constraint on the number of remote neighbors whereas switches of remote for local nodes occur only if the local ones are faster. We call this enduser QoS preserving policy Local Only If Faster (LOIF).

- $\delta=1, \mu=\min (W,|V(T)|-1)$ : Again there is no constraint on the number of remote neighbors but local nodes are preferred independently of their speed comparison to remotes. This is the standard Locality we introduced in Sect. 3.

- $\delta=1, \mu=1$ : As before all switches of remotes for locals are performed. Of the remaining remotes only one is retained and the rest are discarded from the neighborhood. We call this policy Strict.

\section{IMPACT OF LOCALITY ON ISPS \& USERS}

The bounds presented in Sect. 3 provide a broad view of the impact of locality on the transit traffic of hundreds of ISPs. They do not, however, provide any information regarding the impact of locality on end user download rates. Earlier work [26, 6] has demonstrated some "win-win" cases in which ISPs benefit by reducing their transit traffic, while at the same time their users get faster download rates. In general, however, talking mostly to local nodes can harm a user's download rate by, e.g., depriving it from faster remote seeders and leechers (the latter can provide optimistic unchokes). Whether this happens depends on the interplay between demographics and speed. In this section we employ the traffic matrix computation methodology of Sect. 5 to present detailed case studies of the impact of different overlay construction mechanisms from Sect. 6 on ISPs and their users. We are primarily interested in discovering the boundaries of the win-win region from locality for both ISPs and users as well as the reasons behind them.

\subsection{Experimental methodology}

Next we present the common parts of our methodology that appear in all experiments. Experiment-specific parts appear in the corresponding sections.

\subsubsection{Input to the experiments}

Demographics: We used the BitTorrent demand demographics measurements presented in Sect. 4. If not otherwise stated, our default dataset will be mn40K.

Speed distributions: If not otherwise stated, we assign to an ISP the median uplink speed of its country [2]. We also use speeds from iPlane 19. One important point is that these represent last mile bottlenecks. We consider network bottlenecks later in Sect. 8 using an experimental prototype and live torrents.

Seeder/leecher ratios: In dataset pb600 we know exactly if a peer is seeder or leacher but in $\mathrm{mn} 40 \mathrm{~K}$ and $\mathrm{mn} 3 \mathrm{~K}$ we do not have this information. To solve this problem, we obtained from the correspondent tracker the number of seeders and leechers for each torrent. Then we made a client in our dataset a seeder with probability equal to the seeder/leecher ratio of its torrent. Thus although we don't have the exact identities of seeders, we do match the real seeder/leecher ratios. We validated this technique with the dataset pb600 obtaining minor variation compared to real seeder distributions. The raason for this is that the seeder/leecher ratio is fairly stable across ISPs.

\subsubsection{Traffic matrix computation}

In our experiments we are interested in quantifying the effects of locality biased overlay construction on a "home" ISP A. We perform this as follows.

(1) Using our measured demand demographics we identify the set of clients $V(T)$ for each torrent $T \in T(A)$ downloaded by clients in our home ISP $A$. We construct Random, LOIF, Locality, and Strict overlay graphs among the nodes in $V(T)$ as described in Sect. 6. We select the nodes to be seeders as described in Sect. 7.1.1 and assume that they perform proportional seeding.

(2) We feed each overlay graph resulting from the combination of the demographics of a torrent $T$ and an overlay construction algorithm, together with an uplink speed distribution to the BitTorrent traffic matrix 


\begin{tabular}{l|l|l|l|} 
ISP & LOIF & Locality & Strict \\
\hline US1 & $\mathbf{3 2 . 0 0 \%}$ & $\mathbf{5 5 . 6 3 \%}$ & $\mathbf{9 7 . 4 7 \%}$ \\
US2 & $28.47 \%$ & $48.40 \%$ & $97.25 \%$ \\
US3 & $26.04 \%$ & $41.45 \%$ & $97.02 \%$ \\
EU1 & $\mathbf{1 0 . 5 0 \%}$ & $\mathbf{3 9 . 1 2 \%}$ & $\mathbf{9 6 . 4 1 \%}$ \\
EU2 & $11.34 \%$ & $44.89 \%$ & $95.95 \%$ \\
EU3 & $16.18 \%$ & $35.57 \%$ & $96.98 \%$
\end{tabular}

(a) Transit traffic reduction under $\mathrm{mn} 40 \mathrm{~K}$ and Ookla speeds.

\begin{tabular}{l|l|l|l|} 
ISP & LOIF & Locality & Strict \\
\hline US1 & $\mathbf{3 4 . 0 3 \%}$ & $\mathbf{7 7 . 8 6 \%}$ & $\mathbf{9 9 . 1 0 \%}$ \\
US2 & $30.56 \%$ & $69.20 \%$ & $98.73 \%$ \\
US3 & $37.11 \%$ & $78.70 \%$ & $99.27 \%$ \\
EU1 & $\mathbf{1 5 . 2 5 \%}$ & $\mathbf{7 2 . 8 0 \%}$ & $\mathbf{9 9 . 3 5 \%}$ \\
EU2 & $21.22 \%$ & $72.26 \%$ & $99.18 \%$ \\
EU3 & $26.57 \%$ & $71.92 \%$ & $99.05 \%$
\end{tabular}

(c) Transit traffic reduction under pb600 and Ookla speeds.

\begin{tabular}{l|l|l|l|} 
ISP & LOIF & Locality & Strict \\
\hline US1 & $\mathbf{- 6 . 7 1 \%}$ & $\mathbf{- 1 . 3 2 \%}$ & $\mathbf{2 . 8 8 \%}$ \\
US2 & $-5.22 \%$ & $-0.83 \%$ & $4.43 \%$ \\
US3 & $-5.74 \%$ & $-1.27 \%$ & $4.96 \%$ \\
EU1 & $\mathbf{- 1 . 4 7 \%}$ & $\mathbf{3 . 3 3 \%}$ & $\mathbf{1 8 . 5 9 \%}$ \\
EU2 & $-0.55 \%$ & $6.35 \%$ & $11.72 \%$ \\
EU3 & $-3.21 \%$ & $2.28 \%$ & $14.67 \%$
\end{tabular}

(b) Degradation of median QoS under mn40K and Ookla speeds.

\begin{tabular}{l|l|l|l|} 
ISP & LOIF & Locality & Strict \\
\hline US1 & $\mathbf{1 6 . 1 4 \%}$ & $\mathbf{5 2 . 1 2 \%}$ & $\mathbf{9 6 . 6 3 \%}$ \\
US2 & $8.77 \%$ & $46.73 \%$ & $95.68 \%$ \\
US3 & $9.18 \%$ & $39.55 \%$ & $94.66 \%$ \\
EU1 & $\mathbf{3 . 9 4 \%}$ & $\mathbf{4 3 . 8 9 \%}$ & $\mathbf{9 4 . 9 2 \%}$ \\
EU2 & $5.68 \%$ & $50.89 \%$ & $94.69 \%$ \\
EU3 & $12.68 \%$ & $41.63 \%$ & $95.62 \%$
\end{tabular}

(d) Transit Traffic Reduction under mn40K and iPlane speeds.

Table 2: Results for ISPs EU1-EU3, US1-US3, under different demographic and speed datasets

computation methodology detailed in Sect. 5. The outcome is a traffic matrix indicating the transmission rate between any two nodes $v, u \in V(T)$.

(3) We adopt a simplified version of routing according to which all traffic between clients of our home ISP and an ISP of the same country goes over unpaid peering links, whereas traffic between clients of our home ISP and another ISP in a different country goes over a paid transit link. This simplified routing is actually on the conservative side, since it reduces the amount of traffic going to the transit link and thus also the potential gains from applying locality.

Repeating steps (1)-(3) for all torrents in $T(A)$ we obtain the aggregate amount of traffic going to the transit link of $A$ due to the torrents appearing in our dataset.

\subsubsection{Performance metrics}

We study two performance metrics. The first one, transit traffic reduction compared to random is of interest to the home ISP. It is defined as follows: ( (aggregate transit under Random)-(aggregate transit under Locality $(\delta, \mu))$ ) / (aggregate transit under Random). The second one, user QoS reduction is of interest to the clients of the home ISP. It is defined as follows: ( $q_{x}$ (download rate under Random)- $q_{x}$ (download rate under Locality $(\delta, \mu))) / q_{x}($ download rate under Random), where $q_{x}$ denotes the $x$-percentile of download rate computed over all nodes of home ISP. If not otherwise stated we will use the median $(x=0.5)$.

\subsection{Comparing overlays}

In Table 2(a) we present the transit traffic reduction under various locality policies with respect to Random for the 6 largest ISPs (3 from Europe and 3 from US) across our different datasets using uplink speeds from [2]. In Tables 2(b) we present the corresponding impact on user QoS. We have obtained similar results for several other ISPs. We will comment mainly based on the ISPs, EU1 and US1, introduced earlier in Sect. 4.5.1.

\subsubsection{Without bounding the number of inter-AS links}

We begin with "mild" locality policies that do not enforce constraints on the number of remote neighbors. The mildest of all, LOIF, replaces remote with local nodes in the neighborhood only if the locals are faster. In the case of US1 this yields a transit traffic reduction of $32 \%$ compared to Random. The corresponding value for EU1 is 10.5\%. US1 is faster than EU1 and performs more switches of remotes for locals under LOIF and thus gets a higher reduction of transit traffic. Looking at Table 2(b) we see that US1 pays no penalty in terms of QoS reduction for the end users from LOIF. Actually, the median value gets a slight speedup indicated by negative values (see Appendix ?? for other percentiles). The situation for EU1 is similar. The preservation of at least the same user QoS is an inherent characteristic of LOIF which by default leads to a win-win situation for both ISPs and users. The transit savings of LOIF can 
however be small, as in the case of EU1.

We can reduce the transit traffic further by imposing a less strict switching rule. Locality switches any remote client with a local one independently of speed. This increases the savings for US1 to $55.63 \%$ compared to Random whereas the corresponding number for EU1 rises to $39.12 \%$. This is the highest transit reduction that can be expected without limiting the number of inter-AS overlay links. This additional transit traffic reduction does not impose any QoS penalty on the customers of US1. EU1 customers on the other hand pay a small reduction of QoS of 3.33\% since they loose some faster remote neighbors (EU1 is not among the fastest ISPs according to the country speeds depicted in Fig. 4). Under Locality win-win is not guaranteed but rather it depends on speed and demographics. For US1 Locality is again a clear win-win whereas for EU1 is almost win-win.

\subsubsection{Unlocalizable torrents}

In the aforementioned results the transit traffic reduction toped at around 55\%. This happens because the demographics of both US1 and EU1 include a long tail of torrents with very few local nodes. These torrents are "unlocalizable" in the sense that all overlay links for them will have to cross the transit link if the corresponding clients are to be given the standard number of neighbors according to BitTorrent's bootstrapping process (40-50 depending on version). The unlocalizable torrents put rigid limits on the transit reduction that can be achieved without enforcing constraints on the number of allowed inter-AS overlay links. Interesting, although the unlocalizable torrents create most of the transit traffic, they are requested by a rather small percentage of the nodes of an ISP. In US1 $90 \%$ of transit traffic under Locality is due to only $10 \%$ of the nodes. In EU1 $90 \%$ of transit traffic is due to $13.44 \%$ of nodes.

\subsubsection{Bounding the number on inter-AS overlay links}

If we want further transit traffic reductions then we need to control the unlocalizable torrents by enforcing strict constraints on the number of inter-AS overlay links. In the last column of Table 2(a) we depict the performance of Strict that permits up to 1 inter-AS overlay link per torrent for a given client. Indeed in this case the transit traffic reduction is huge (around 96\%$97 \%$ for both networks). The median user QoS drops by $18.59 \%$ in EU1. The situation is much better for US1 where the median speed drops by around $3 \%$. However, nodes that are downloading unlocalizable torrents pay a heavy penalty of almost $99 \%$.

\subsection{Comparing ISPs}

Inspecting Table 2(a) we see that American ISPs in general achieve higher transit traffic reduction than Eu- ropean ones, across all locality biased overlay construction policies. We attribute this to the fact that Random performs very poor in those ISPs since their content is more scattered around the world (they have smaller Inherent Localizability, Sect.4.5.1). When comparing among American or among European ISPs, the observed differences correlate mostly with the size of the ISP. The reason is that in ISPs with approximately the same Inherent Localizability (e.g., the 3 American ISPs), Random performs approximately the same, and thus any difference in transit reduction comes from the performance of Locality or LOIF. The latter depend on the absolute size of the ISP since a larger ISP can gather more easily enough local peers to reach the minimum number required by the bootstrapping process.

\section{VALIDATION ON LIVE TORRENTS}

In our study up to now, we have only considered last mile bottlenecks imposed by access link speeds but no network bottlenecks due to congestion or ISP traffic engineering, including throttling [9]. In addition, although we have evaluated the accuracy of $b$-matching in a controlled emulation environment (Appendix B), we can obtain further insights by testing our results in the wild where we can observe additional effects from delays and losses that are lacking from an emulation environment that captures only bandwidths. To address such issues we integrated LOIF, Locality, and Strict $(\mu)$ into the mainline Bittorrent client (version 5.2.2). Next we describe briefly some implementation issues and then move to present the results of connecting to live torrents with our modified client.

\subsection{Prototype implementation}

Integrating these policies into the existing BitTorrent clients requires addressing some new requirements. First, we need to know for every Bittorrent client its ISP and country. For this, we use the MaxMind geolocation database [1. Next, we need to discover the list of local clients in order to be able to substitute remote ones with local ones. For this, we use the PEX messages to discover all the participants of the swarm, and then use the MaxMind database to classify them. Finally, the last requirement which is specific to LOIF, is to estimate the speed of the local and remote clients. For this, we monitor the rate at which the clients send us HAVE messages, which indicates how fast they download. Finally, notice that the above method works only for inferring the speed of leechers. For seeders, we can only infer the speed of those seeders that unchoke us. Thus in LOIF we do not switch neighbors for which we do not have speed information.

Next, we briefly describe our implementation of LOIF. Many implementation decisions are influenced by how 


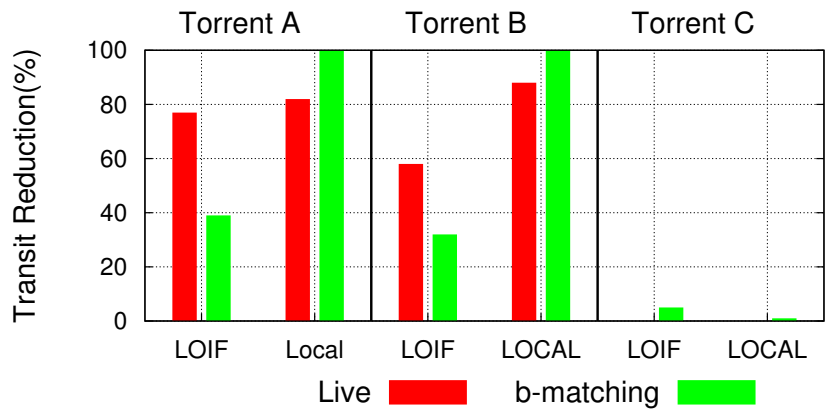

Figure 6: Comparision between LOIF and Local

\begin{tabular}{cccc}
\hline & Local & Remote & Percentage of Seed \\
\hline Torrent A & 521 & 46 & $63.2 \%$ \\
Torrent B & 351 & 211 & $12.5 \%$ \\
Torrent C & 3 & 666 & $66.4 \%$ \\
\hline
\end{tabular}

\section{Table 3: Live torrent characteristics}

the python mainline Bittorrent client is designed and implemented. Every 40 seconds we perform the following steps:

- We classify the active neighbor peers in 3 lists: $L_{I S P}$ which contains all peers that belong to the same ISP as the client, $L_{\text {Peering }}$ which contains all peers that are in ISPs with peering relationships and $L_{\text {Remote }}$ which contains other peers.

- For every peer $R_{i} \in L_{\text {Remote }}$, we close the connection if there exists a peer $L_{j} \in L_{I S P}$ with higher estimated download speed. If such a peer does not exist then we check if there exists a peer $C_{j} \in L_{\text {Peering }}$ with higher estimated download speed and in this case again we close the connection 2

- For each connection that was closed in the last step, the algorithm opens a new one, giving preference, first to those IPs that belong to the same ISP, then to those IPs belonging to peering ISPs and, finally, to those IPs belonging to other ISPs.

An important detail in our implementation is to always have a minimum number of neighbors (at least 40). This holds for LOIF and Locality, but not for Strict. For Strict $(\mu)$, we close connections and don't open new ones, if we have more than $\mu$ remote nodes.

\subsection{Experimental methodology}

We ran our modified Bittorrent client from an ADSL connection of ISP EU1. In all the torrents we first warmed up by downloading 30MB to avoid BitTorrent's

\footnotetext{
${ }^{2}$ The assumption is that nodes in the same country communicate over peering links. In our implementation we do not infer ISP relationships but we can do so with iPlane Nano 20].
}

startup phase. In each run, we re-initialize back to the same 30MB. Next, we download 50MB with each of the following policies: Random, LOIF, Locality, and Strict. We repeated each test 5 times, and reported averages over all runs. During each experiment we logged the list of IPs and the number of neighbors and used them later as input to our traffic matrix estimation technique of Sect. 5. This way, we can compare the estimated transit savings with the real one on live torrents.

\subsection{High, medium, and low localizability tor- rents}

We used our prototype to validate some of our previous results. Although we cannot scale to the number of torrents discussed in Sect. 7, we tested torrents at characteristic points of the demographic spectrum. In particular, we tested a very popular torrent inside EU1 (Torrent A), an intermediately popular one (Torrent B), and an unpopular one (Torrent C). In Table 3 we summarize the characteristics of the 3 torrents. In Fig. 6 we present the transit traffic savings as predicted by our traffic matrix estimation method and as measured on the live torrent under LOIF and Locality. We do not present results under Strict as they were always in perfect agreement.

Overall we see that the results under Locality are pretty consistent - estimation and measurement are within $10-20 \%$ of each other. In terms of absolute values things are as expected: in cases A and B there are enough local nodes to eliminate almost all transit traffic whereas in $\mathrm{C}$ there is 0 saving as there do not exist any local nodes to switch to. The difference between the $100 \%$ savings predicted by $b$-matching in A and B and the ones measured in practice has to do with implementation restrictions. As mentioned earlier, we update the overlay every $40 \mathrm{sec}$ (which is equal to 4 unchoke intervals). During that time new incoming remote connections are accepted and can lead to unchokes that create transit traffic and thus eat away from the $100 \%$ saving expected upon overlay update instants when all remote connections are switched with local ones.

Under LOIF, the deviation between estimation and measurement is substantial: the measured transit saving is twice as big as the estimated one. To interpret this, we looked at the number of switches of remote nodes for local ones that LOIF performed in practice and realized that they were much more than we would predict. This in effect means that the real LOIF found the remote nodes to be slower than what expected from our speed dataset from Ookla [2. We attribute this to network bottlenecks or throttling at the inter-AS links of EU1 or the ISPs that host the remote nodes. Although certainly interesting, identifying exactly why the remote nodes appear slower than expected is beyond the scope of the current work. See [9] for more. 


\section{RELATED WORK}

\subsection{Early work on locality-biasing}

One of the early works on locality-biased overlay construction was Karagiannis et al. 14. Using traces from a campus network as well as a six-month-long logfile from a popular torrent, they showed that there is substantial overlap in the torrents downloaded by co-located clients. Another early work from Bindal et al. [4, studied the effect of limiting the number of inter-AS connections using simulations with synthetic demand. Aggarwal et al. 3. studied the effects of locality biasing on the Gnutella overlay. Apart from studying a different $\mathrm{P} 2 \mathrm{P}$ system, they differ from our work by focusing on the overlay graph theoretic properties whereas we care about the traffic matrix.

\subsection{Recent systems for locality-biasing}

Following up on positive results on the potential of locality-biasing, a number of actual systems like P4P [26] and ONO [6] have appeared recently for the BitTorrent $\mathrm{P} 2 \mathrm{P}$ protocol. The previous works focus on architectural and systems questions regarding "how" to implement locality-biasing, and in particular whether the goal can be achieved through unilateral client-only solutions, or bilateral cooperation is essential for making locality work for both ISPs and users. In terms of reported results, 26 presents a variety of use cases for $\mathrm{P} 4 \mathrm{P}$ over different networks and $\mathrm{P} 2 \mathrm{P}$ applications like Pando and Liveswarms. In all cases however, results are based on one or a few swarms and thus do not capture the aggregate effects created by tens of thousands of concurrent swarms with radically different demographics. The results reported in [6] on the other hand, are indeed from multiple torrents and networks, but they only report on the final outcome from using the ONO system without explaining how the demographics of the torrents and the speeds of the ISPs affect these outcomes. The main driving force behind our work is to explain "when" locality works and "why" when it does so and thus help in interpreting the results from systems like $\mathrm{P} 4 \mathrm{P}$ and $\mathrm{ONO}$ or others to come in the future. Locality biasing has also been applied to $\mathrm{P} 2 \mathrm{P}$ streaming systems [23].

\subsection{BitTorrent measurements}

A substantial amount of work has gone into BitTorrent measurements [13, 12, 24, 21. These works go beyond locality to characterize things like the arrival pattern of new nodes, the seeding duration, the seeder/leecher ratios, etc. Our work apart from performing large scale measurements develops scalable methodologies that permit distilling non-trivial conclusions regarding the interplay of demographics, speed, and overlay construction. Relevant to our work is the recent work of Piatek et al. 22]. It discusses the potential for win-win outcomes for ISPs and users but puts most of its emphasis on implementation issues and the consequences of strategically behaving ISPs. Our work, on the other hand, is of performance evaluation nature and aims at pushing the envelope in terms of both the scalability and the fidelity of our evaluation methodology. Our dataset is large; we compute transit reduction from our entire $40 \mathrm{~K}$ of torrents whereas they use only 1000 torrents out of their $20 \mathrm{~K}$ dataset. In terms of methodology, we capture the effect of stratification from choke/unchoke whereas [22] assumes cooperative clients and does not model the effect of speed.

\section{CONCLUSIONS}

In this paper we collected extensive measurements of real BitTorrent demand demographics and developed scalable methodologies for computing their resulting traffic matrix. Based on this we quantified the impacts of different locality-biasing overlay construction algorithms on ISPs and end-users. By studying several real ISPs, we have shown that locality yields win-win situations in most cases. The win-win profile is bounded by "unlocalizable" torrents that have few local neighbors. Handling the unlocalizable torrents requires limiting the number of allowed inter-AS overlay connections. This has a small impact on the average user but a dire one on the users of unlocalizable torrents.

\section{REFERENCES}

[1] MaxMind- GeoIP. info at http://www.maxmind.com/app/ip-location

[2] Ookla's speedtest throughput measures. https://confluence.slac.stanford.edu/display/IEPM/Ookla's+Speedtes

[3] Vinay Aggarwal, Anja Feldmann, and Christian Scheideler. Can ISPs and P2P users cooperate for improved performance? ACM SIGCOMM Comput. Commun. Rev., 37(3):29-40, 2007.

[4] R. Bindal, P. Cao, W. Chan, J. Medved, G. Suwala, T. Bates, and A. Zhang. Improving traffic locality in BitTorrent via biased neighbor selection. In ICDCS'06.

[5] Katarína Cechlárová and Tamás Fleiner. On a generalization of the stable roommates problem. ACM Trans. Algorithms, 1(1):143-156, 2005.

[6] David R. Choffnes and Fabián E. Bustamante. Taming the torrent: a practical approach to reducing cross-isp traffic in peer-to-peer systems. In Proc. of ACM SIGCOMM '08.

[7] Bram Cohen. Incentives build robustness in BitTorrent. In Proc. of First Workshop on Economics of Peer-to-Peer Systems, Berkeley, CA, USA, Jun 2003.

[8] Marcel Dischinger, Andreas Haeberlen, Krishna P. Gummadi, and Stefan Saroiu. Characterizing residential broadband networks. In Proc. of ACM IMC 'O\%.

[9] Marcel Dischinger, Alan Mislove, Andreas Haeberlen, and Krishna P. Gummadi. Detecting bittorrent blocking. In Proc. ACM IMC'08.

[10] W. Feller. An Introduction to Probability Theory and Its Applications. Wiley, New York, 1968.

[11] Anh-Tuan Gai, Fabien Mathieu, Fabien de Montgolfier, and Julien Reynier. Stratification in p2p networks: Application to bittorrent. In Proc. of ICDCS'O\%.

[12] L. Guo, S. Chen, Z. Xiao, E. Tan, X. Ding, and X. Zhang. Measurements, analysis, and modeling of bittorrent-like 
systems. In Proc. of ACM IMC'05.

[13] Mikel Izal, Guillaume Urvoy-Keller, Ernst W Biersack, Pascal A Felber, Anwar Al Hamra, and Luis Garces-Erice. Dissecting bittorrent: Five months in a torrent's lifetime. In Proc. of PAM '04.

[14] Thomas Karagiannis, Pablo Rodriguez, and Konstantina Papagiannaki. Should internet service providers fear peer-assisted content distribution? In Proc. ACM IMC'05.

[15] Nikolaos Laoutaris, Damiano Carra, and Pietro Michiardi Uplink allocation beyond choke/unchoke or how to divide and conquer best. In Proc. of ACM CoNEXT'08.

[16] A. Legout, G. Urvoy-Keller, and P. Michiardi. Rarest first and choke algorithms are enough. In Proc. of ACM IMC'06.

[17] Arnaud Legout, Nikitas Liogkas, Eddie Kohler, and Lixia Zhang. Clustering and sharing incentives in bittorrent systems. In Proc. of ACM SIGMETRICS '0\%.

[18] Tom Leighton. Improving performance on the internet. Commun. ACM, 2009.

[19] Harsha V. Madhyastha, Tomas Isdal, Michael Piatek, Colin Dixon, Thomas Anderson, Arvind Krishnamurthy, and Arun Venkataramani. iPlane: An information plane for distributed services. In OSDI, 2006.

[20] Harsha V. Madhyastha, Ethan Katz-Bassett, Thomas Anderson, Arvind Krishnamurthy, and Arun Venkataramani. iPlane Nano: Path prediction for Peer-to-Peer applications. In Proc. of NSDI'09.

[21] D. Menasche, A. Rocha, B. Li, D. Towsley, and A. Venkataramani. Content availability in swarming systems: Models, measurements and bundling implications. In Proc. of ACM CoNEXT'O9.

[22] Michael Piatek, Harsha V. Madhyastha, John P. John, Arvind Krishnamurthy, and Thomas Anderson. Pitfalls for ISP-friendly P2P design. In Proc. of HotNets-VIII.

[23] Fabio Picconi and Laurent Massoulié. ISP friend or foe? making P2P live streaming ISP-aware. In Proc. of IEEE ICDCS'09.

[24] J.A. Pouwelse, P. Garbacki, D.H.J. Epema, and H.J. Sips. The BitTorrent P2P file-sharing system: Measurements and analysis. In Proc. of IPTPS'05.

[25] Jimmy J. M. Tan. A necessary and sufficient condition for the existence of a complete stable matching. J. Algorithms, 12(1):154-178, 1991.

[26] Haiyong Xie, Yang Richard Yang, Arvind Krishnamurthy, Yanbin Liu, and Avi Silberschatz. P4P: Provider portal for applications. In Proc. of ACM SIGCOMM'08.

\section{APPENDIX}

\section{A. MODELING REGULAR UNCHOKES WITH} A $B$-MATCHING

The input to a $b$-matching problem consists of a set of nodes $V$, and functions $n: V \rightarrow 2^{V}, b: V \rightarrow \mathbb{Z}^{+}$, and $p: V^{2} \rightarrow \mathbb{R}^{+}$defined as follows: $n(v)$ defines the set of nodes to which $v$ can be matched with (matching is symmetric here, and thus $u \in n(v)$ iff $v \in n(u)$ ); $b(v)$ defines the number of parallel matchings that $v$ is allowed to establish; $p(v, u)$ is a measure of the preference that $v$ has for becoming stably matched to $u$. A solution to a $b$-matching is a set $M$ of matchings (edges) between pairs of nodes in $V$, such that for each matched pair $(v, u) \in M$, the matching and capacity constraints $n, b$ are satisfied and further, there exists no "blocking pair" $\left(v^{\prime}, u^{\prime}\right) \in M$, i.e., no pair that satisfies: $p\left(v, v^{\prime}\right)>p(v, u)$ and $p\left(v^{\prime}, v\right)>p\left(v^{\prime}, u^{\prime}\right)$.

It is easy to see that there exists a direct mapping from BitTorrent to $b$-matching [11. Looking at a particular node $v$ and torrent $T$ : the neighborhood $N(v, T)$ can be mapped to the allowed matchings $n(v)$; the number of parallel unchokes $k$ (default value for $k$ being 4 ) at each $10 \mathrm{sec}$ interval corresponds to $b(v)$, the number of matchings allowed for $v$; the uplink capacity $U(v)$ of a BitTorrent client $v$ can be used as a measure of the preference $p(u, v)$ that each node $u \neq v$ would have for being matched with $v$ in the context of a $b$-matching. $b$ matchings in which the preference for a node is the same independently of who is considering, i.e., for given $u$, $p(v, u)=p\left(v^{\prime}, u\right), \forall v, v^{\prime}$, are said to have a global preference function. Tan 25] has shown that the existence of a stable solution for the $b$-matching problem relates to the non-existence of circles in the preference function $p$, which is a condition that is certainly satisfied under a global preference function like $U(v)$. Therefore, for the aforementioned mapping from BitTorrent to $b$-matching, one can use a simple $O(|V(T)| \cdot k)$ greedy algorithm to find the unique stable matching that exists in this case 11,3

\section{B. VALIDATION OF MODELING}

In this section we validate the accurately of modeling the unchoke algorithm using a $b$-matching. We look at the two typical phases of a torrent's lifetime [24].

\section{B.1 Startup phase}

During the initial phase of a new torrent leechers hold few chunks and thus whether two nodes unchoke each other depends, beyond their speeds, on the set of chunks they hold. The $b$-matching modeling of unchoke described in Sect. 5.2 assumes that steady-state has been reached and thus chunk (in)availability does not affect the resulting matchings. In Appendix [C] we extend this basic matching algorithm to allow it to also capture the completion level $c(v)$ of a node, i.e., the percent-

age of a file of total size $C$ that it holds. We have used this completion-level aware $b$-matching in conjunction with small initial completion levels $c(v)<1 \%$ for all leechers to estimate the effect chunk availability on the aggregate capacity of a torrent. BitTorrent's LRF chunk selection strategy is used for expediting the convergence to steady state. We verified this by comparing our two implementations of $b$-matching. In Fig. 7 we give an indicative example to show that for everything but very small files, the startup phase is much shorter than steady state. For this reason we can ignore it at small cost in terms of accuracy and focus on the baseline $b$-matching that is more scalable to large datasets than the more complicated one involving completion levels.

\footnotetext{
${ }^{3}$ Uniqueness is guaranteed under the assumption that there are not ties in speeds. We made sure that his is the case by adding to our speed datasets a very small random noise.
} 


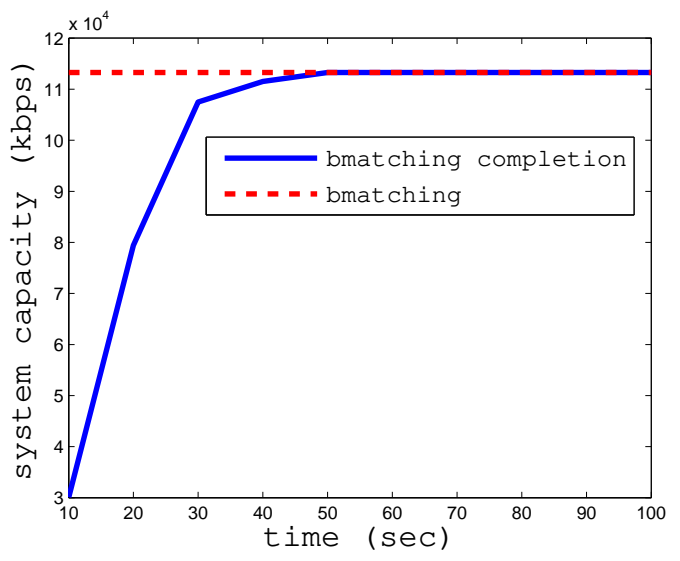

Figure 7: Aggregate system capacity from baseline $b$-matching and $b$-matching with completion levels. Parameters: $|V|=40$, uplink rates randomly distributed with mean 2 Mbps, $C=10000$, $c_{0}(v)<1 \%, \forall v$, unchoke duration=10 sec, chunk size $=32$ kBytes. The more complex version converges within a minute to the steady-state value predicted by the baseline $b$-matching. Download completion requires around $\mathbf{3 0}$ mins.

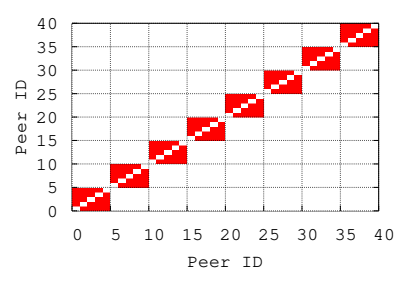

(a) b-matching Unchoking (b) Emulation Unchoking

Figure 8: Unchoking Patterns

\section{B.2 Steady state}

Next we validate the extent at which the steady state matchings predicted by $b$-matching resemble the unchoking behavior of an actual mainline client of BT (v.3.4.2) running in a controlled emulation environment. More specifically, we set-up a dummy torrent with 40 clients, the slowest of which, was given an uplink capacity of $80 \mathrm{Kbps}$, whereas successive ones were made increasingly faster using a step of $24 \mathrm{Kbps}$. We chose such a small increment to recreate a rather difficult environment for stratification [17] to arise. For each client, we measured the frequency at which the client unchokes each other client, and then we put a mark on Fig. 8(b) for the $k$ clients that it unchokes more frequently (client ids on the figure are assigned according to upload bandwidth in increasing order). Comparing with Fig. 8(a) that depicts the same information from the execution of $b$-matching under the same uplink capacities, it is easy to see that $b$-matching provides a reasonable prediction of the real unchokes that take place and therefore can be used as a scalable tool for processing huge numbers of small and large torrents that would otherwise be impossible to simulate concurrently. We got similar accuracy using many other torrent sizes and uplink distributions, including empirical ones from measurement.

\section{COMPLETION LEVEL AWARE $B$-MATCHING}

\section{C.1 Edge filtering}

Let $c(v)$ denote the number of chunks already downloaded by node $v$ out of the total $C$ chunks that make up a complete file. For a pair of neighbors $(v, u)$ with $c(v) \geq c(u)$ let $I(v \rightarrow u), c(v)-c(u) \leq I(v \rightarrow u) \leq c(v)$ denote the number of chunks of $v$ that "are of interest" to $u$, i.e., chunks that $v$ has downloaded but $u$ hasn't. It is easy to see that $I(u \rightarrow v)=c(u)-c(v)+I(v \rightarrow u)$, $0 \leq I(u \rightarrow v) \leq c(u)$. If we assume that the chunks held at some point in time by a node are a random subset of the entire set of chunks, which is reasonable granted LRF [7], then it follows that:

$$
\begin{aligned}
p_{v u}(x) & =P\{I(v \rightarrow u)=x, I(u \rightarrow v)=c(u)-c(v)+x\} \\
& =H y p e r G e o(c(u)-x, c(v), C, c(u))
\end{aligned}
$$

where HyperGeo( $d, p, s, s s)$ denotes a hyper geometric pmf [10] giving the probability of drawing $d$ "successes" with a sample of size $s s$ from a pool of $p$ items, of which $s$ are "successes". Then the expected amount of interest in the two directions is:

$$
\begin{aligned}
& E\{I(v \rightarrow u)\}=\sum_{x=c(v)-c(u)}^{c(v)} x \cdot p_{v u}(x) \\
& E\{I(u \rightarrow v)\}=\sum_{x=c(v)-c(u)}^{c(v)}(c(u)-c(v)+x) \cdot p_{v u}(x)
\end{aligned}
$$

For pair $(v, u)$ we define its filtering probability to be:

$\phi(v, u)=\min \left(\frac{E\{I(v \rightarrow u)\}}{T \cdot U(v) \cdot(\sigma \cdot k)^{-1}}, \frac{E\{I(u \rightarrow v)\}}{T \cdot U(u) \cdot(\sigma \cdot k)^{-1}}, 1\right)$

where $\sigma$ is the size of a chunk and $T$ is the duration of an unchoke interval. Given an instance of a $b$ matching problem $\langle V, n, b, p\rangle$ we filter it to obtain a new one $\left\langle V, n^{\prime}, b, p\right\rangle$ in which we keep an edge $(v, u)$, meaning that $v \in n(u), u \in n(v)$ and $v \in n^{\prime}(u), u \in n^{\prime}(v)$, with probability $\phi(v, u)$, whereas we drop it with probability $1-\phi(v, u)$. 


\section{C.2 Time-evolving completion ratios}

Let $c_{t}(v)$ be the completion ratio of node $v$ at time $t$ and let $M_{t}$ be the stable matching obtained from solving the $b$-matching $\left\langle V, n^{\prime}, b, p\right\rangle$ in which $n^{\prime}$ is obtained from $n$ after applying the filtering procedure of Sect.C.1 with completion ratios $\left\{c_{t}(v): v \in V\right\}$. Then the completion ratios of nodes can be updated at the end of the unchoke interval as follows:

$c_{t+T}(v)=c_{t}(v)+\sum_{u:(v, u) \in M_{t}} \min \left(E\{I(u \rightarrow v)\}, \frac{T \cdot U(u)}{\sigma \cdot k}\right)$

Thus with the above we have a method for mapping the effects of completion levels on the state of a torrent and consequently on the resulting matchings. 